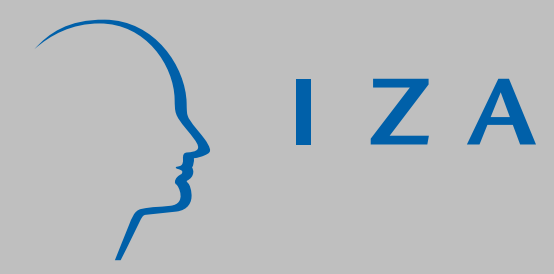

IZA DP No. 703

New Wine in Old Bottles: A Sequential Estimation Technique for the LPM

William C. Horrace

Ronald L. Oaxaca

January 2003 


\title{
New Wine in Old Bottles: A Sequential Estimation Technique for the LPM
}

\author{
William C. Horrace \\ Syracuse University
}

Ronald L. Oaxaca

University of Arizona and IZA Bonn

Discussion Paper No. 703
January 2003

IZA

P.O. Box 7240

D-53072 Bonn

Germany

Tel.: +49-228-3894-0

Fax: +49-228-3894-210

Email: iza@iza.org

This paper can be downloaded without charge at: http://ssrn.com/abstract=383102

An index to IZA Discussion Papers is located at: http://www.iza.org/publications/dps/

This Discussion Paper is issued within the framework of IZA's research area Evaluation of Labor Market Policies and Projects. Any opinions expressed here are those of the author(s) and not those of the institute. Research disseminated by IZA may include views on policy, but the institute itself takes no institutional policy positions.

The Institute for the Study of Labor (IZA) in Bonn is a local and virtual international research center and a place of communication between science, politics and business. IZA is an independent, nonprofit limited liability company (Gesellschaft mit beschränkter Haftung) supported by the Deutsche Post AG. The center is associated with the University of Bonn and offers a stimulating research environment through its research networks, research support, and visitors and doctoral programs. IZA engages in (i) original and internationally competitive research in all fields of labor economics, (ii) development of policy concepts, and (iii) dissemination of research results and concepts to the interested public. The current research program deals with (1) mobility and flexibility of labor, (2) internationalization of labor markets, (3) welfare state and labor market, (4) labor markets in transition countries, (5) the future of labor, (6) evaluation of labor market policies and projects and (7) general labor economics.

IZA Discussion Papers often repre sent preliminary work and are circulated to encourage discussion. Citation of such a paper should account for its provisional character. A revised version may be available on the IZA website (www.iza.org) or directly from the author. 
IZA Discussion Paper No. 703

January 2003

\section{ABSTRACT \\ New Wine in Old Bottles: \\ A Sequential Estimation Technique for the LPM*}

The conditions under which ordinary least squares (OLS) is an unbiased and consistent estimator of the linear probability model (LPM) are unlikely to hold in many instances. Yet the LPM still may be the correct model or a good approximation to the probability generating process. A sequential least squares (SLS) estimation procedure is introduced that may outperform OLS in terms of finite sample bias and yields a consistent estimator. Monte Carlo simulations reveal that SLS outperforms OLS, probit and logit in terms of mean squared error of the predicted probabilities.

JEL Classification: $\quad$ C25

Keywords: linear probability model, sequential least squares, consistency, Monte Carlo

Corresponding author:

Ronald L. Oaxaca

Room 401QQ

401 W. McClelland Hall

University of Arizona

P.O. Box 210108

Tucson, AZ 85721-0108

Tel.: +15206214135

Email: rlo@email.arizona.edu

\footnotetext{
* We gratefully acknowledge valuable comments by Seung Ahn, Badi Baltagi, Dan Houser, Price Fishback, Art Gonzalez, Shawn Kantor, Alan Ker, Paul Ruud and Peter Schmidt. Capable research assistance was provided by Nidhi Thakur.
} 


\section{Introduction}

The limitations of the Linear Probability Model (LPM) are well known. Estimated probabilities based on OLS are not necessarily bounded on the unit interval and unbiased (OLS) estimation implies that heteroscedasticity is present. Conventional textbook advice points to probit or logit as the standard remedy. These models bound the maximum likelihood estimated probabilities on the unit interval. However, the fact that consistent estimation of the LPM may be difficult does not imply that either probit or logit is the correct specification of the probability model. In some cases it is reasonable to assume that probabilities are generated from bounded linear decision rules. Theoretical rationalizations for the LPM can be found in Rosenthal (1989) and Heckman and Snyder (1997). Despite the attractiveness of the logit and probit specifications for modeling a binary dependent variable as a function of covariates, the LPM remains a venerable model in the literature. OLS estimation of the LPM is still used as an empirical tool for its computational parsimony and ease of coefficient interpretation. Recent applications of the LPM include Lukashin (2000), Fairlie and Sundstrom (1999), Lucking-Reiley (1999), and Currie and Gruber (1996).

Some well-known LPM theorems are provided in Amemiya (1977), and the quintessential survey on binary dependent variables is Amemiya (1981). Standard econometrics textbooks, such as Greene (2000) and Kmenta (1997), point out LPM modelling complications that can lead to biased and inconsistent OLS estimates. Nevertheless, the literature is not sufficiently clear on the precise conditions under which OLS estimation yields problematic estimators of the parameters of the LPM. The purpose of this paper is: a) to rigorously lay out these conditions; b) to derive the finite-sample and asymptotic biases of OLS when they are present; and c) to provide additional results that shed light on the appropriateness or inappropriateness of OLS estimation of the LPM. Moreover, this paper proposes a consistent sequential estimation strategy that is functionally simpler than probit and logit in the sense that numerical optimization 
is not required to produce the LPM estimates. Since the estimator is based on a simple bias correction for OLS, the coefficients estimates produced are the true marginal effects and do not need to be transformed to yield readily interpretable results (as is the case with logit and probit).

The plan of the paper is as follows. Section 2 provides a few theorems and results from OLS estimation of the LPM that have, heretofore, not been rigorously presented. Section 3 introduces a sequential estimation technique, Sequential Least Squares (SLS), for the LPM that is simple to implement. Section 4 presents a simulation study that compares results from the new sequential estimator to those from OLS and finds that the sequential estimator outperforms OLS in terms of finite sample bias. Section 5 performs a simulation study demonstrating that the sequential estimator outperforms probit and logit when the underlying data generation process is LPM. The metric of performance is the mean squared error of the predicted probabilities of the dependent variable. Section 6 is a summary and conclusion.

\section{LPM Specification and Main Results}

A general way in which to specify the Data Generating Process (DGP) for the LPM is as follows. Let $y_{i}$ be a binary random variable that takes on the values 0 or 1 . Let $x_{i}$ be a continuous random $1 \times k$ vector of explanatory variables on $\Re^{k}, \beta$ be a $k \times 1$ vector of coefficients, and $\varepsilon_{i}$ be an unobserved random error term. For convenience we will define the following probabilities over the random variable $x_{i} \beta \in \Re$.

$$
\begin{aligned}
& \operatorname{Pr}\left(x_{i} \beta>1\right)=\pi, \\
& \operatorname{Pr}\left(x_{i} \beta \in[0,1]\right)=\gamma, \\
& \operatorname{Pr}\left(x_{i} \beta<0\right)=\rho,
\end{aligned}
$$

where $\pi+\gamma+\rho=1$. Consider a random sample of data: $\left(y_{i}, x_{i}\right) ; i \in N ; N=\{1, \ldots, n\}$. 
In what follows it will be useful to introduce the following notation. Define the sets

$$
\begin{aligned}
& \kappa_{\gamma}=\left\{i \mid x_{i} \beta \in[0,1]\right\}, \\
& \kappa_{\pi}=\left\{i \mid x_{i} \beta>1\right\} .
\end{aligned}
$$

which are the sets of all indices $i$ such that $x_{i} \beta$ is on the unit interval and of all indices $i$ such that $x_{i} \beta>1$, respectively. Notice that $\kappa_{\gamma}, \kappa_{\pi} \subseteq N, \kappa_{\gamma} \cap \kappa_{\pi}=\emptyset$. Equation (1) implies

$$
\begin{aligned}
& \operatorname{Pr}\left(i \in \kappa_{\pi}\right)=\pi, \\
& \operatorname{Pr}\left(i \in \kappa_{\gamma}\right)=\gamma, \\
& \operatorname{Pr}\left(i \notin \kappa_{\gamma} \cup \kappa_{\pi}\right)=\rho .
\end{aligned}
$$

Let the values of $y_{i}$ be generated according to:

$$
\begin{aligned}
y_{i} & =1 \text { for } i \in \kappa_{\pi}, \\
& =x_{i} \beta+\varepsilon_{i} \text { for } i \in \kappa_{\gamma}, \\
& =0 \text { otherwise. }
\end{aligned}
$$

(As convention dictates, we will assume the first element of the vector $x_{i}$ is always a 1 , so that the first element of $\beta$ is an intercept term.) The conditional probability function for $y_{i}$ is then

$$
\begin{aligned}
& \operatorname{Pr}\left(y_{i}=1 \mid x_{i}, i \in \kappa_{\pi}\right)=1, \\
& \operatorname{Pr}\left(y_{i}=1 \mid x_{i}, i \in \kappa_{\gamma}\right)=x_{i} \beta, \\
& \operatorname{Pr}\left(y_{i}=0 \mid x_{i}, i \in \kappa_{\gamma}\right)=1-x_{i} \beta, \\
& \operatorname{Pr}\left(y_{i}=1 \mid x_{i}, i \notin \kappa_{\gamma} \cup \kappa_{\pi}\right)=0 .
\end{aligned}
$$

Therefore, $y_{i}$ traces the familiar ramp function on $x_{i} \beta$, which can be thought of as the cumulative distribution function of a continuous uniform random variable on $[0,1]$. 
The DGP specification then implies the following error process:

$$
\begin{aligned}
\varepsilon_{i} & =0 \text { for } i \in \kappa_{\pi}, \\
& =y_{i}-x_{i} \beta, i \in \kappa_{\gamma}, \\
& =0 \text { for } i \notin \kappa_{\gamma} \cup \kappa_{\pi} .
\end{aligned}
$$

Notice that $\varepsilon_{i}$ is not binary and is realized with the following conditional probabilities

$$
\begin{aligned}
& \operatorname{Pr}\left(\varepsilon_{i}=0 \mid x_{i}, i \in \kappa_{\pi}\right)=1, \\
& \operatorname{Pr}\left(\varepsilon_{i}=1-x_{i} \beta \mid x_{i}, i \in \kappa_{\gamma}\right)=x_{i} \beta, \\
& \operatorname{Pr}\left(\varepsilon_{i}=-x_{i} \beta \mid x_{i}, i \in \kappa_{\gamma}\right)=1-x_{i} \beta, \\
& \operatorname{Pr}\left(\varepsilon_{i}=0 \mid x_{i}, i \notin \kappa_{\gamma} \cup \kappa_{\pi}\right)=1 .
\end{aligned}
$$

Estimation of the LPM typically proceeds by OLS on the model:

$$
y_{i}=x_{i} \beta+u_{i}, i \in N,
$$

where it is assumed that $u_{i}$ is a zero-mean random variable that is independent of the $x_{i}$. The resulting estimator will be problematic as proven in the sequel. Notice that OLS specifies an error term $u_{i}$, which is different than $\varepsilon_{i}$ :

$$
\begin{aligned}
u_{i} & =1-x_{i} \beta \text { for } i \in \kappa_{\pi}, \\
& =y_{i}-x_{i} \beta \text { for } i \in \kappa_{\gamma}, \\
& =-x_{i} \beta \text { for } i \notin \kappa_{\gamma} \cup \kappa_{\pi} .
\end{aligned}
$$

The conditional probability function for $u_{i}$ is

$$
\begin{aligned}
& \operatorname{Pr}\left(u_{i}=1-x_{i} \beta \mid x_{i}, i \in \kappa_{\pi}\right)=1, \\
& \operatorname{Pr}\left(u_{i}=1-x_{i} \beta \mid x_{i}, i \in \kappa_{\gamma}\right)=x_{i} \beta, \\
& \operatorname{Pr}\left(u_{i}=-x_{i} \beta \mid x_{i}, i \in \kappa_{\gamma}\right)=1-x_{i} \beta, \\
& \operatorname{Pr}\left(u_{i}=-x_{i} \beta \mid x_{i}, i \notin \kappa_{\gamma} \cup \kappa_{\pi}\right)=1 .
\end{aligned}
$$


It is extremely important in what follows to distinguish between $u_{i}$, the OLS error, and $\varepsilon_{i}$, the error of the true DGP, for it is this distinction that induces problems with OLS of the LPM. Figure 1 illustrates this distinction: the first panel is the DGP for the conditional mean function, the second panel is the LPM error $\left(\varepsilon_{i}\right)$, and the third panel is the OLS error $\left(u_{i}\right)$. Notice that $u_{i}$ can assume two different values: 1$x_{i} \beta$ and $-x_{i} \beta$, while $\varepsilon_{i}$ can assume three: $0,1-x_{i} \beta$ and $-x_{i} \beta$. The conditional probability functions of equations (4), (5) and (7) imply the following conditional expectations

$$
\begin{aligned}
& E\left(y_{i} \mid x_{i}, i \in \kappa_{\pi}\right)=1, \\
& E\left(y_{i} \mid x_{i}, i \in \kappa_{\gamma}\right)=x_{i} \beta, \\
& E\left(y_{i} \mid x_{i}, i \notin \kappa_{\gamma} \cup \kappa_{\pi}\right)=0, \\
& E\left(\varepsilon_{i} \mid x_{i}, i \in \kappa_{\pi}\right)=0, \\
& E\left(\varepsilon_{i} \mid x_{i}, i \in \kappa_{\gamma}\right)=0, \\
& E\left(\varepsilon_{i} \mid x_{i}, i \notin \kappa_{\gamma} \cup \kappa_{\pi}\right)=0, \\
& E\left(u_{i} \mid x_{i}, i \in \kappa_{\pi}\right)=1-x_{i} \beta, \\
& E\left(u_{i} \mid x_{i}, i \in \kappa_{\gamma}\right)=0, \\
& E\left(u_{i} \mid x_{i}, i \notin \kappa_{\gamma} \cup \kappa_{\pi}\right)=-x_{i} \beta .
\end{aligned}
$$

The expectations make clear the obvious difference between $u_{i}$ and $\varepsilon_{i}$ : $u_{i}$ only has zero-mean when $i \in \kappa_{\gamma} ; \varepsilon_{i}$ always has zero-mean. This is also intuitively obvious in Figure 1.

Theorem 1 If $\gamma<1$, Ordinary Least Squares Estimation of the Linear Probability Model is generally biased and inconsistent. 
Proof. The conditional expectation of the usual OLS error is

$$
\begin{aligned}
& E\left(u_{i} \mid x_{i}, i \in \kappa_{\pi}\right)=1-x_{i} \beta, \\
& E\left(u_{i} \mid x_{i}, i \in \kappa_{\gamma}\right)=0, \\
& E\left(u_{i} \mid x_{i}, i \notin \kappa_{\gamma} \cup \kappa_{\pi}\right)=-x_{i} \beta .
\end{aligned}
$$

Therefore, the conditional expectation of the OLS error, $u_{i}$, is a function of $x_{i}$ with probability $(1-\gamma)$. Hence, OLS will be biased and inconsistent, if $\gamma<1$.

The proof formalizes the specification error made when the OLS conditional mean is assumed. This fact has been mentioned by a few authors but has never be stated with any probabilistic rigor. The upshot of the theorem is that only those observations in the set $\kappa_{\gamma}$ have a well-behaved error associated with them, so OLS that includes any observations outside of the set $\kappa_{\gamma}$ will be problematic. In what follows we present a few additional results, derive the finite and asymptotic sample biases, and suggest a bias reduction method for OLS based on a sequential estimation strategy.

Remark 2 If $\kappa_{\gamma} \neq N$, OLS estimation is biased and inconsistent. That is, if the sample used to estimate $\beta$ contains any $i \notin \kappa_{\gamma}$, then $\gamma$ is necessarily less than 1 , so OLS is problematic.

Of course the entire problem is due to $\gamma<1$, so the follow is not surprising.

Remark 3 If $\gamma=1, O L S$ is unbiased and consistent, because $\pi=\rho=0, E\left(u_{i} \mid\right.$ $\left.x_{i}\right)=0$ for all $i \in N$, and the conditional expectation function implied by the DGP is:

$$
E\left(y_{i} \mid x_{i}\right)=\operatorname{Pr}\left(y_{i}=1 \mid x_{i}\right)=x_{i} \beta, i \in N
$$

Therefore the usual OLS results hold under suitable regularity conditions. 
Define discrete random variables $z_{i}$ and $w_{i}$ where:

$$
\begin{aligned}
z_{i} & =1 \text { for } i \in \kappa_{\gamma} \\
& =0 \text { otherwise. } \\
w_{i} & =1 \text { for } i \in \kappa_{\pi}, \\
& =0 \text { otherwise. }
\end{aligned}
$$

So, $\operatorname{Pr}\left(z_{i}=1\right)=\gamma$ and $\operatorname{Pr}\left(w_{i}=1\right)=\pi$. Then an alternative specification of the DGP in equation (3) is:

$$
y_{i}=w_{i}+z_{i} x_{i} \beta+u_{i} z_{i} ; \quad i \in N
$$

This specification is convenient, because it makes explicit the fact that $u_{i}$ is not the correct error term associated with the DGP, instead $\varepsilon_{i}=u_{i} z_{i}$ is correct. It will also be useful in the sequel. Notice,

$$
\begin{aligned}
u_{i} z_{i} & =0 \text { for } i \notin \kappa_{\gamma}, \\
& =1-x_{i} \beta \text { for } y_{i}=1, i \in \kappa_{\gamma} \\
& =-x_{i} \beta \text { for } y_{i}=0, i \in \kappa_{\gamma} .
\end{aligned}
$$

Moreover, the conditional probability function for $u_{i} z_{i}$ is the same as $\varepsilon_{i}$ :

$$
\begin{aligned}
& \operatorname{Pr}\left(u_{i} z_{i}=0 \mid x_{i}, i \in \kappa_{\pi}\right)=1, \\
& \operatorname{Pr}\left(u_{i} z_{i}=1-x_{i} \beta \mid x_{i}, i \in \kappa_{\gamma}\right)=x_{i} \beta, \\
& \operatorname{Pr}\left(u_{i} z_{i}=-x_{i} \beta \mid x_{i}, i \in \kappa_{\gamma}\right)=1-x_{i} \beta, \\
& \operatorname{Pr}\left(u_{i} z_{i}=0 \mid x_{i}, i \notin \kappa_{\gamma} \cup \kappa_{\pi}\right)=1 .
\end{aligned}
$$

It is evident that $E\left(u_{i} z_{i} \mid x_{i}\right)=0$, so the specification in equation (9) has a zero-mean 
error, which is independent of $x_{i}$. Taking the unconditional mean of equation (9):

$$
\begin{aligned}
E\left(y_{i}\right) & =\pi+E\left(z_{i} x_{i}\right) \beta+E\left(u_{i} z_{i}\right) \\
& =\pi+\gamma E\left(z_{i} x_{i} \mid z_{i}=1\right) \beta+\gamma E\left(z_{i} u_{i} \mid z_{i}=1\right) \\
& =\pi+\gamma E\left(x_{i} \mid z_{i}=1\right) \beta+\gamma E\left(u_{i} \mid z_{i}=1\right) \\
& =\pi+\gamma \mu_{x \gamma} \beta
\end{aligned}
$$

where

$$
\begin{aligned}
\mu_{x \gamma} \beta & =E\left(x_{i} \mid z_{i}=1\right) \beta \\
& =E\left(x_{i} \beta \mid z_{i}=1\right) \\
& =\int_{0}^{1} x_{i} \beta f\left(x_{i} \beta \mid z_{i}=1\right) d\left(x_{i} \beta\right) \\
& =\frac{1}{\gamma} \int_{0}^{1} x_{i} \beta f\left(x_{i} \beta\right) d\left(x_{i} \beta\right),
\end{aligned}
$$

and $f\left(x_{i} \beta \mid z_{i}=1\right)$ is the bounded conditional probability density and $f\left(x_{i} \beta\right)$ is the bounded marginal probability density of $x_{i} \beta$. Since $0<\mu_{x \gamma} \beta<1$, and $E\left(y_{i}\right)$ is a weighted average of $1, \mu_{x \gamma} \beta$, and 0 , it follows that $0<E\left(y_{i}\right)<1$. The mean function of equation (10) will be used in the sequel. Consider the OLS estimator:

$$
\widehat{\beta}_{n}=\left[\sum_{i \in N} x_{i}^{\prime} x_{i}\right]^{-1} \sum_{i \in N} x_{i}^{\prime} y_{i} .
$$

Substituting equation (9):

$$
\widehat{\beta}_{n}=\left[\sum_{i \in N} x_{i}^{\prime} x_{i}\right]^{-1} \sum_{i \in N} x_{i}^{\prime}\left(w_{i}+z_{i} x_{i} \beta+u_{i} z_{i}\right) .
$$

The data can be partitioned into those $i \in \kappa_{\gamma}$, those $i \in \kappa_{\pi}$ and those that are in neither subset. Taking into consideration the values of $z_{i}$ and $w_{i}$ in these three 
regimes:

$$
\begin{aligned}
\widehat{\beta}_{n} & =\left[\sum_{i \in N} x_{i}^{\prime} x_{i}\right]^{-1}\left[\sum_{i \notin \kappa_{\gamma} \cup \kappa_{\pi}} x_{i}^{\prime}(0)+\sum_{i \in \kappa_{\gamma}} x_{i}^{\prime}\left(x_{i} \beta+u_{i}\right)+\sum_{i \in \kappa_{\pi}} x_{i}^{\prime}(1)\right] \\
& =\left[\sum_{i \in N} x_{i}^{\prime} x_{i}\right]^{-1}\left[\sum_{i \in \kappa_{\gamma}} x_{i}^{\prime} x_{i} \beta+\sum_{i \in \kappa_{\gamma}} x_{i}^{\prime} u_{i}+\sum_{i \in \kappa_{\pi}} x_{i}^{\prime}\right]
\end{aligned}
$$

Taking expectations conditional on $x_{i}$ :

$$
\begin{aligned}
E\left(\widehat{\beta}_{n} \mid x_{i}\right) & =\left[\sum_{i \in N} x_{i}^{\prime} x_{i}\right]^{-1}\left[\sum_{i \in \kappa_{\gamma}} x_{i}^{\prime} x_{i} \beta+\sum_{i \in \kappa_{\gamma}} x_{i}^{\prime} E\left(u_{i} \mid x_{i}, \quad i \in \kappa_{\gamma}\right)+\sum_{i \in \kappa_{\pi}} x_{i}^{\prime}\right] \\
& =\left[\sum_{i \in N} x_{i}^{\prime} x_{i}\right]^{-1}\left[\sum_{i \in \kappa_{\gamma}} x_{i}^{\prime} x_{i} \beta+\sum_{i \in \kappa_{\gamma}} x_{i}^{\prime}(0)+\sum_{i \in \kappa_{\pi}} x_{i}^{\prime}\right] \\
E\left(\widehat{\beta}_{n} \mid x_{i}\right) & =\left[\sum_{i \in N} x_{i}^{\prime} x_{i}\right]^{-1} \sum_{i \in \kappa_{\gamma}} x_{i}^{\prime} x_{i} \beta+\left[\sum_{i \in N} x_{i}^{\prime} x_{i}\right]_{i \in \kappa_{\pi}} x_{i}^{\prime} \neq \beta,
\end{aligned}
$$

which is generally biased because $\gamma<1$. The bias will persist asymptotically. When $\gamma=1, \kappa_{\gamma}=N$, the first term on the RHS reduces to $\beta$, the second term on the RHS goes to 0 , and $\widehat{\beta}_{n}$ is unbiased.

The inconsistency of $\widehat{\beta}_{n}$ follows in a similar fashion. Letting $C$ denote the cardinality operator, define $n_{\pi}=C\left(\kappa_{\pi}\right), n_{\gamma}=C\left(\kappa_{\gamma}\right)$ and $n_{\rho}=n-n_{\pi}-n_{\gamma}$. Let plim denote the probability limit operator as $n \rightarrow \infty$. Assume $\operatorname{plim}\left[n^{-1} \sum_{i \in N} x_{i}^{\prime} x_{i}\right]=Q$ and $\operatorname{plim}\left[n_{\gamma}^{-1} \sum_{i \in \kappa_{\gamma}} x_{i}^{\prime} x_{i}\right]=Q_{\gamma}$ where $Q$ and $Q_{\gamma}$ are finite, (non singular) positive definite. Assume $\operatorname{plim}\left[n_{\pi}^{-1} \sum_{i \in \kappa_{\pi}} x_{i}^{\prime}\right]=\mu_{x \pi}^{\prime}, \operatorname{plim}\left[n^{-1} \sum_{i \in N} x_{i}^{\prime}\right]=\mu_{x}^{\prime}$ and $\operatorname{plim}\left[n_{\gamma}^{-1} \sum_{i \in \kappa_{\gamma}} x_{i}^{\prime} u_{i}\right]=0$, where $\mu_{x \pi}^{\prime}$ and $\mu_{x}^{\prime}$ are finite vectors. Assume $\operatorname{plim}\left[n^{-1} n_{\pi}\right]=$ $\pi$ and $\operatorname{plim}\left[n_{\gamma} n^{-1}\right]=\gamma$. Then it is easy to show that

$$
\operatorname{plim}\left(\widehat{\beta}_{n}\right)=Q^{-1}\left(Q_{\gamma} \beta \gamma+\pi \mu_{x \pi}^{\prime}\right) \neq \beta
$$

Equation (12) seems to imply that even if $\gamma$ and $\pi$ were known, $\widehat{\beta}_{n}$ could not be bias-corrected. Yet, the unconditional mean of $y_{i}$ in equation (10) seems to imply 
that if $\gamma$ and $\pi$ were known, an OLS regression of $\left(y_{i}-\pi\right)$ on $\left(\gamma x_{i}\right)$ might produce an unbiased estimate. Define the OLS estimator from such a transformed regression as:

$$
\widehat{\beta}_{n}^{*}=\left[\sum_{i \in N} \gamma^{2} x_{i}^{\prime} x_{i}\right]^{-1} \sum_{i \in N} \gamma x_{i}^{\prime}\left(y_{i}-\pi\right) .
$$

Theorem $4 \widehat{\beta}_{n}^{*}$ is biased and inconsistent for $\beta$.

Proof. After some algebra, equation (13) implies

$$
\begin{aligned}
\widehat{\beta}_{n}^{*} & =\frac{1}{\gamma}\left[\sum_{i \in N} x_{i}^{\prime} x_{i}\right]^{-1} \sum_{i \in N} x_{i}^{\prime} y_{i}-\frac{1}{\gamma}\left[\sum_{i \in N} x_{i}^{\prime} x_{i}\right]^{-1} \sum_{i \in N} x_{i}^{\prime} \pi \\
& =\frac{1}{\gamma} \hat{\beta}_{n}-\frac{\pi}{\gamma}\left[\sum_{i \in N} x_{i}^{\prime} x_{i}\right]^{-1} \sum_{i \in N} x_{i}^{\prime} .
\end{aligned}
$$

Taking expectations

$$
\begin{aligned}
E\left(\widehat{\beta}_{n}^{*} \mid x_{i}\right)= & \frac{1}{\gamma} E\left(\hat{\beta}_{n} \mid x_{i}\right)-\frac{\pi}{\gamma}\left[\sum_{i \in N} x_{i}^{\prime} x_{i}\right]^{-1} \sum_{i \in N} x_{i}^{\prime} . \\
= & \frac{1}{\gamma}\left\{\left[\sum_{i \in N} x_{i}^{\prime} x_{i}\right]^{-1} \sum_{i \in \kappa_{\gamma}} x_{i}^{\prime} x_{i} \beta+\left[\sum_{i \in N} x_{i}^{\prime} x_{i}\right]^{-1} \sum_{i \in \kappa_{\pi}} x_{i}^{\prime}\right\} \\
& -\frac{\pi}{\gamma}\left[\sum_{i \in N} x_{i}^{\prime} x_{i}\right]^{-1} \sum_{i \in N} x_{i}^{\prime} \\
\neq & \beta .
\end{aligned}
$$

Thus, knowledge of $\pi$ and $\gamma$ to estimate $E\left(y_{i} \mid x_{i}\right)$ by $O L S$ does not in general lead to an unbiased estimator of $\beta$. Moreover it does not lead to consistent estimation by OLS:

$$
\begin{aligned}
\operatorname{plim}\left(\widehat{\beta}_{n}^{*}\right) & =\frac{1}{\gamma} \operatorname{plim}\left(\hat{\beta}_{n}\right)-\frac{\pi}{\gamma}\left[\operatorname{plim}\left(n^{-1} \sum_{i \in N} x_{i}^{\prime} x_{i}\right)\right]^{-1} \operatorname{plim}\left(n^{-1} \sum_{i \in N} x_{i}^{\prime}\right) \\
& =\frac{1}{\gamma}\left[Q^{-1}\left(Q_{\gamma} \beta \gamma+\mu_{x \pi}^{\prime} \pi\right)\right]-\frac{\pi}{\gamma} Q^{-1} \mu_{x}^{\prime} \\
& =Q^{-1}\left[Q_{\gamma} \beta+\left(\mu_{x \pi}^{\prime}-\mu_{x}^{\prime}\right) \frac{\pi}{\gamma}\right] \neq \beta .
\end{aligned}
$$


The bias will persist asymptotically. The problem with the estimators $\widehat{\beta}_{n}$ and $\widehat{\beta}_{n}^{*}$ is not that $\gamma$ and $\pi$ are unknown but that $\kappa_{\gamma}$ is unknown, for if we knew $\kappa_{\gamma}$, we could perform OLS only on those observations therein contained.

Remark 5 Therefore, sufficient information for unbiased and consistent OLS estimation is knowledge of $\kappa_{\gamma}$.

Also notice that if $\kappa_{\gamma}=N$, then:

$$
\sum_{i \in \kappa_{\gamma}} x_{i}^{\prime} x_{i}=\sum_{i \in N} x_{i}^{\prime} x_{i} \text {, and } \sum_{i \in \kappa_{\pi}} x_{i}^{\prime}=0
$$

Therefore, equation (12), reduces to:

$$
\begin{aligned}
E\left(\widehat{\beta}_{n} \mid x_{i}\right) & =\left[\sum_{i \in N} x_{i}^{\prime} x_{i}\right]^{-1} \sum_{i \in N} x_{i}^{\prime} x_{i} \beta+\left[\sum_{i \in N} x_{i}^{\prime} x_{i}\right]^{-1} 0, \\
E\left(\widehat{\beta}_{n} \mid x_{i}\right) & =\beta
\end{aligned}
$$

unbiased for $\kappa_{\gamma}=N$. A similar argument can be made to show the consistency of this estimate. Of course if $\gamma=1$, then $\kappa_{\gamma}=N$.

Remark 6 Therefore, without knowledge of $\kappa_{\gamma}$ and $\kappa_{\pi}$, a sufficient condition for unbiased OLS estimation when $\gamma<1$ is $\kappa_{\gamma}=N$.

$\kappa_{\gamma}=N$ is a weaker sufficient condition than $\gamma=1$, but probably unlikely in reasonably large samples. For any given random sample $\left(y_{i}, x_{i}\right) ; i \in N$, the $\operatorname{Pr}\left[\kappa_{\gamma}=\right.$ $N]=\gamma^{n}$, so

$$
\lim _{n \rightarrow \infty} \operatorname{Pr}\left[\kappa_{\gamma} \neq N\right]=\lim _{n \rightarrow \infty}\left(1-\gamma^{n}\right)=1 .
$$

Remark 7 Therefore, without knowledge of $\kappa_{\gamma}$ and $\kappa_{\pi}$, if $\gamma<1$ and $\kappa_{\gamma}=N$, then as $n \rightarrow \infty, \kappa_{\gamma} \neq N$ with probability approaching 1 , and $\widehat{\beta}_{n}$ is asymptotically biased and inconsistent. 
It should be noted that as the sample size grows, once the first observation $x_{i} \beta \notin$ $[0,1]$ appears in $N$ then $\kappa_{\gamma} \neq N$ and finite sample unbiasedness is lost also. Oddly

enough the estimator $\widehat{\beta}_{n}$ could, under the right conditions, be reliable in small samples and unreliable in large samples. If we had knowledge of the sets $\kappa_{\gamma}$ and $\kappa_{\pi}$, then a consistent estimate of $\beta$ could be based on the sub-sample:

$$
\begin{gathered}
\widehat{\beta}_{\kappa_{\gamma}}=\left[\sum_{i \in \kappa_{\gamma}} x_{i}^{\prime} x_{i}\right]^{-1} \sum_{i \in \kappa_{\gamma}} x_{i}^{\prime} y_{i}, \text { for } \kappa_{\gamma} \text { and } \kappa_{\pi} \text { known, } \\
\widehat{\beta}_{\kappa_{\gamma}}=\left[\sum_{i \in \kappa_{\gamma}} x_{i}^{\prime} x_{i}\right]^{-1} \sum_{i \in \kappa_{\gamma}} x_{i}^{\prime}\left(w_{i}+z_{i} x_{i} \beta+u_{i} z_{i}\right), \\
\widehat{\beta}_{\kappa_{\gamma}}=\left[\sum_{i \in \kappa_{\gamma}} x_{i}^{\prime} x_{i}\right]^{-1} \sum_{i \in \kappa_{\gamma}} x_{i}^{\prime}\left(x_{i} \beta+u_{i}\right), \\
E\left(\widehat{\beta}_{\kappa_{\gamma}} \mid x_{i}\right)=\beta, \text { for } \kappa_{\gamma} \text { known. }
\end{gathered}
$$

This is tantamount to removing the observations $i \notin \kappa_{\gamma}$. Then a consistent estimate of $\gamma$ is $\widehat{\gamma}=C\left(\kappa_{\gamma}\right) / n$, and a consistent estimate of $\pi$ is $\widehat{\pi}=C\left(\kappa_{\pi}\right) / n$.

\section{Sequential Least Squares}

The beauty of OLS estimation of the LPM is that it is an extremely simple procedure. Therefore any modifications or corrections to the OLS estimator need to be themselves simple, if one is interested in salvaging the model in any meaningful way. One can certainly envision myriad sophisticated estimators that would be an improvement over OLS: an MLE technique that estimated $\gamma$ and $\pi$ as well as $\beta$, a non-linear search algorithm that recognizes the constraint $x_{i} \beta \in[0,1]$, some sort of splines technique that estimates the $x_{i} \beta=0$ and $x_{i} \beta=1$ break points, etc.. However, these would not be a substitute for OLS insofar as they are not simple procedures. We now present a simple OLS correction technique. 
If somehow the observations $i \notin \kappa_{\gamma}$ could be eliminated sequentially, then as the elimination sequence grew: $N$ would decrease to some set of observations that was a subset of $\kappa_{\gamma}$, while $\kappa_{\pi} \rightarrow \emptyset$, then $\widehat{\beta}_{n}$ would converge in some probabilistic sense to $\beta$. Therefore, an empirical strategy could involve finding a $\widehat{\beta}_{i \in \kappa_{\gamma}}$ estimate that ensures that the predicted dependent variable is on the unit interval. One specific estimation strategy is to identify the empirical subsets

$$
\begin{aligned}
\widehat{\kappa}_{\gamma}^{(1)} & =\left\{i \mid i \in N \cap x_{i} \widehat{\beta}_{n} \in[0,1]\right\}, \\
\widehat{\kappa}_{\pi \cup \rho}^{(1)} & =\left\{i \mid i \in N \cap x_{i} \widehat{\beta}_{n} \notin[0,1]\right\},
\end{aligned}
$$

with cardinality $C\left(\widehat{\kappa}_{\gamma}^{(1)}\right)=n_{\gamma}^{(1)} \leq n$ and $C\left(\widehat{\kappa}_{\pi \cup \rho}^{(1)}\right)=n_{\pi \cup \rho}^{(1)} \leq n$. Then a subsample estimate of $\beta$ is obtained as

$$
\tilde{\beta}_{i \in \widehat{\kappa}_{\gamma}^{(1)}}=\left[\sum_{i \in \widehat{\kappa}_{\gamma}^{(1)}} x_{i}^{\prime} x_{i}\right]^{-1} \sum_{i \in \widehat{\kappa}_{\gamma}^{(1)}} x_{i}^{\prime} y_{i}
$$

Define the subset

$$
\begin{aligned}
\widehat{\kappa}_{\gamma}^{(2)} & =\left\{i \mid i \in \widehat{\kappa}_{\gamma}^{(1)} \cap x_{i} \tilde{\beta}_{i \in \widehat{\kappa}_{\gamma}^{(1)}} \in[0,1]\right\}, \\
\widehat{\kappa}_{\pi \cup \rho}^{(2)} & =\left\{i \mid i \in \widehat{\kappa}_{\gamma}^{(1)} \cap x_{i} \tilde{\beta}_{i \in \widehat{\kappa}_{\gamma}^{(1)}} \notin[0,1]\right\},
\end{aligned}
$$

with cardinality $C\left(\widehat{\kappa}_{\gamma}^{(2)}\right)=n_{\gamma}^{(2)} \leq n_{\gamma}^{(1)}$ and $C\left(\widehat{\kappa}_{\pi \cup \rho}^{(2)}\right)=n_{\pi \cup \rho}^{(2)} \leq n_{\gamma}^{(1)}$. Then a second subsample estimate is

$$
\tilde{\beta}_{i \in \widehat{\kappa}_{\gamma}^{(2)}}=\left[\sum_{i \in \widehat{\kappa}_{\gamma}^{(2)}} x_{i}^{\prime} x_{i}\right]_{i \in \widehat{\kappa}_{\gamma}^{(2)}} x_{i}^{\prime} y_{i}
$$

We can repeat this process in general:

$$
\tilde{\beta}_{i \in \widehat{\kappa}_{\gamma}^{(j)}}=\left[\sum_{i \in \widehat{\kappa}_{\gamma}^{(j)}} x_{i}^{\prime} x_{i}\right]^{-1} \sum_{i \in \widehat{\kappa}_{\gamma}^{(j)}} x_{i}^{\prime} y_{i}, j=1, \ldots, J
$$


for

$$
\begin{aligned}
\widehat{\kappa}_{\gamma}^{(j)} & =\left\{i \mid i \in \widehat{\kappa}_{\gamma}^{(j-1)} \cap x_{i} \tilde{\beta}_{i \in \widehat{\kappa}_{\gamma}^{(j-1)}} \in[0,1]\right\}, \\
\widehat{\kappa}_{\pi \cup \rho}^{(j)} & =\left\{i \mid i \in \widehat{\kappa}_{\gamma}^{(j-1)} \cap x_{i} \tilde{\beta}_{i \in \widehat{\kappa}_{\gamma}^{(j-1)}} \notin[0,1]\right\}, j=2, \ldots, J
\end{aligned}
$$

with cardinality $C\left(\widehat{\kappa}_{\gamma}^{(j)}\right)=n_{\gamma}^{(j)} \leq n_{\gamma}^{(j-1)}$ and $C\left(\widehat{\kappa}_{\pi \cup \rho}^{(j)}\right)=n_{\pi \cup \rho}^{(j)} \leq n_{\gamma}^{(j-1)}$ until convergence in the sense that all the observations in the final subsample satisfy $x_{i} \tilde{\beta}_{i \in \widehat{\kappa}_{\gamma}^{(J)}} \in$ $[0,1]$. The final estimate will be

$$
\tilde{\beta}_{i \in \widehat{\kappa}_{\gamma}^{(J)}}=\left[\sum_{i \in \widehat{\kappa}_{\gamma}^{(J)}} x_{i}^{\prime} x_{i}\right]^{-1} \sum_{i \in \widehat{\kappa}_{\gamma}^{(J)}} x_{i}^{\prime} y_{i} .
$$

Call this the Sequential Least Squares (SLS) estimator. The convergence of the sequential estimators imply

$$
\frac{n_{\gamma}^{(j)}}{n_{\gamma}^{(j-1)}} \rightarrow 1 \text { and } n_{\pi \cup \rho}^{(j)} \rightarrow 0
$$

as $n \rightarrow \infty$ and $j \rightarrow J$. This condition must hold in order for the trimming to converge in any meaningful way, or else the entire sample would ultimately be discarded.

Theorem 8 If prediction error $x_{i}\left(\tilde{\beta}_{i \in \widehat{\kappa}_{\gamma}^{(j)}}-\beta\right)$ is a continuous random variable on $\Re$ and if $\operatorname{Pr}\left\{x_{i} \tilde{\beta}_{i \in \widehat{\kappa}_{\gamma}^{(j)}} \in[0,1]\right\} \rightarrow 1$ as $n \rightarrow \infty$ and as $j \rightarrow J$, then the SLS estimator $\tilde{\beta}_{i \in \widehat{\kappa}_{\gamma}^{(J)}}$ is consistent for $\beta$.

Proof. Appendix available upon request.

It is not entirely clear when the condition $\operatorname{Pr}\left\{x_{i} \tilde{\beta}_{i \in \widehat{\kappa}_{\gamma}^{(j)}} \in[0,1]\right\} \rightarrow 1$ as $n \rightarrow$ $\infty$ and as $j \rightarrow J$ will hold, since the probability in question is a function of the random variable $x_{i}$, whose distribution is unknown, in general. However, Horrace and Oaxaca (2001) show that under certain conditions normality of $x_{i}$ is sufficient to ensure $\operatorname{Pr}\left\{x_{i} \tilde{\beta}_{i \in \widehat{\kappa}_{\gamma}^{(j)}} \in[0,1]\right\} \rightarrow 1$, so the set of possible distributions of $x_{i}$ that 
satisfies the convergence conditions is certainly not empty. In the sequel we perform a simulation study that examines the extent to which the SLS estimator outperforms OLS in terms of sample bias.

Define sets based on the final estimator $\tilde{\beta}_{i \in \widehat{\kappa}_{\gamma}^{(J)}}$ :

$$
\begin{aligned}
\widehat{\kappa}_{\gamma}^{*} & =\left\{i \mid x_{i} \tilde{\beta}_{i \in \widehat{\kappa}_{\gamma}^{(J)}} \in[0,1]\right\}, \\
\widehat{\kappa}_{\pi}^{*} & =\left\{i \mid x_{i} \tilde{\beta}_{i \in \widehat{\kappa}_{\gamma}^{(J)}}>1\right\}, \\
\widehat{\kappa}_{\rho}^{*} & =\left\{i \mid x_{i} \tilde{\beta}_{i \in \widehat{\kappa}_{\gamma}^{(J)}}<0\right\}, \\
\widehat{\kappa}_{\pi \cup \rho}^{*} & =\widehat{\kappa}_{\pi}^{*} \cup \widehat{\kappa}_{\rho}^{*} \\
& =\left\{i \mid x_{i} \tilde{\beta}_{i \in \widehat{\kappa}_{\gamma}^{(J)}} \notin[0,1]\right\} .
\end{aligned}
$$

Notice the difference between $\widehat{\kappa}_{\gamma}^{*}$ and $\widehat{\kappa}_{\gamma}^{(J)}$, and between $\widehat{\kappa}_{\pi \cup \rho}^{*}$ and $\widehat{\kappa}_{\pi \cup \rho}^{(J)}$. $\widehat{\kappa}_{\gamma}^{*}$, and $\widehat{\kappa}_{\pi \cup \rho}^{*}$ are based on the entire sample $N$ and $\widehat{\kappa}_{\gamma}^{(J)}$ and $\widehat{\kappa}_{\pi \cup \rho}^{(J)}$ are based on the subsample $\widehat{\kappa}_{\gamma}^{(J-1)}$. Then insofar as $\tilde{\beta}_{i \in \hat{\kappa}_{\gamma}^{(J)}}$ is consistent for $\beta$, consistent estimates of the probabilities $\gamma, \pi$, and $\rho$ can be obtained from

$$
\begin{aligned}
& \tilde{\gamma}=\frac{C\left(\widehat{\kappa}_{\gamma}^{*}\right)}{n} \\
& \tilde{\pi}=\frac{C\left(\widehat{\kappa}_{\pi}^{*}\right)}{n} \\
& \tilde{\rho}=1-\tilde{\pi}-\tilde{\gamma},
\end{aligned}
$$

Note that if $n_{\gamma}^{(1)}=n$, then no trimming is necessary, $N=\widehat{\kappa}_{\gamma}^{(J)}=\widehat{\kappa}_{\gamma}^{*}$ and $\tilde{\gamma}=$ 1. Clearly, this estimator should only be used if the sample size is large, since observations $x_{i} \beta \in[0,1]$ will be trimmed with positive probability. When the final SLS estimator is used to predict $y_{i}$ we are assured that $\widetilde{y}_{i}=x_{i} \tilde{\beta}_{i \in \widehat{\kappa}_{\gamma}^{(J)}} \in[0,1]$ for $i \in \widehat{\kappa}_{\gamma}^{*}$, however this will not necessarily be the case for all $\widetilde{y}_{i}, i \in N$. As is usually 
the case, prediction of $y_{i}$ can be performed as follows:

$$
\begin{aligned}
& \widetilde{y}_{i}=1 \text { for } x_{i} \tilde{\beta}_{i \in \widehat{\kappa}_{\gamma}^{(J)}}>1 \text { for } i \in N \\
& \widetilde{y}_{i}=x_{i} \tilde{\beta}_{i \in \widehat{\kappa}_{\gamma}^{(J)}} \text { for } x_{i} \tilde{\beta}_{i \in \widehat{\kappa}_{\gamma}^{(J)}} \in[0,1] \text { for } i \in N \\
& \widetilde{y}_{i}=0 \text { for } x_{i} \tilde{\beta}_{i \in \widehat{\kappa}_{\gamma}^{(J)}}<0 \text { for } i \in N .
\end{aligned}
$$

We now present a brief simulation study that demonstrates that the SLS trimming estimator $\tilde{\beta}_{i \in \widehat{\kappa}_{\gamma}^{(J)}}$ is generally less biased that the OLS estimator $\widehat{\beta}_{n}$.

\section{Simulation Study: SLS Versus OLS}

A simulation study was conducted to assess the performance of the SLS estimator against the OLS estimator in terms of finite sample bias. Initially, we are concerned with understanding the nature of the OLS bias when $\gamma<1$ and seeing if the SLS estimator is an improvement over OLS. To assess estimator performance for different values of $\gamma$, requires selecting $\gamma, \pi$ and $\beta$ then finding an appropriate multivariate distribution for the $x_{i}$ to generate data such that $\operatorname{Pr}\left\{x_{i} \beta \in[0,1]\right\}=\gamma$ and $\operatorname{Pr}\left\{x_{i} \beta>\right.$ $1\}=\pi$. For $x_{i}$ with large dimensionality this would be a monumental task, therefore we restrict attention to the bivariate model

$$
\begin{aligned}
y_{i} & =1 \text { for } i \in \kappa_{\pi}, \\
& =\beta_{0}+\beta_{1} x_{i}+\varepsilon_{i} \text { for } i \in \kappa_{\gamma} \\
& =0 \text { otherwise }
\end{aligned}
$$

where $\beta_{0}, \beta_{1}$ and $x_{i}$ are scalars. We also assume that $x_{i}$ has a normal distribution with mean $\mu$ and variance $\sigma$. Given these restrictions on the data generation process, it is a relatively simple procedure to select $\gamma, \pi, \beta_{0}$ and $\beta_{1}$, and then to calculate $\mu$ and $\sigma$, such that $\operatorname{Pr}\left\{\beta_{0}+\beta_{1} x_{i} \in[0,1]\right\}=\gamma$ and $\operatorname{Pr}\left\{\beta_{0}+\beta_{1} x_{i}>1\right\}=\pi$. To generate data in this way, notice that for $\beta_{1}>0$

$$
\operatorname{Pr}\left\{\beta_{0}+\beta_{1} x_{i} \in[0,1]\right\}=\operatorname{Pr}\left\{g_{i} \in[\underline{c}, \bar{c}]\right\}
$$




$$
\begin{aligned}
\underline{c} & =\frac{-\beta_{0}-\beta_{1} \mu}{\beta_{1} \sigma}, \\
\bar{c} & =\frac{1-\beta_{0}-\beta_{1} \mu}{\beta_{1} \sigma},
\end{aligned}
$$

where $g_{i}$ is a standard normal random variate. Given $\gamma, \pi, \beta_{0}$ and $\beta_{1}$, the necessary $\mu$ and $\sigma$ can be calculated by solving

$$
\begin{aligned}
& \Phi^{-1}(1-\gamma-\pi)=\frac{-\beta_{0}-\beta_{1} \mu}{\beta_{1} \sigma}, \\
& \Phi^{-1}(1-\pi)=\frac{1-\beta_{0}-\beta_{1} \mu}{\beta_{1} \sigma},
\end{aligned}
$$

where $\Phi^{-1}$ is the inverse cumulative distribution function of a standard normal random variate. That is,

$$
\begin{aligned}
\mu & =-\frac{\beta_{0}}{\beta_{1}}-\frac{\Phi^{-1}(1-\gamma-\pi)}{\beta_{1}\left[\Phi^{-1}(1-\pi)-\Phi^{-1}(1-\gamma-\pi)\right]} \\
\sigma & =\frac{1}{\beta_{1}\left[\Phi^{-1}(1-\pi)-\Phi^{-1}(1-\gamma-\pi)\right]}
\end{aligned}
$$

As a practical matter, the sample size, $n$, must be fairly large to ensure that the SLS procedure doesn't trim the entire sample, (i.e. $\left.\widehat{\kappa}_{\gamma}^{(J)}=\emptyset\right)$. Since the SLS estimator trims realizations $x_{i} \beta \in[0,1]$ with positive probability, if there are only a few of these observations in a small sample, then they may all be trimmed during the procedure. Large $n$ is not an unreasonable restriction to impose on the study, since the SLS estimator should only be used in situations where the sample size is fairly large. Therefore we select sample sizes of $n=500,1000$ and 2000. Simulation iterations were arbitrarily set at 100 to calculate the empirical bias of the OLS and SLS estimates of $\beta_{0}$ and $\beta_{1}$. Parameter values were arbitrarily selected as $\gamma=0.25$ and $0.75, \pi=$ $0.10,0.20, \beta_{0}=-0.50,0.50$, and $\beta_{1}=1.00,2.00$. Results are contained in Tables 1 4. In all tables the OLS estimators are $\widehat{\beta}_{0}$ and $\widehat{\beta}_{1}$, while the SLS estimators are $\tilde{\beta}_{0}$ and $\tilde{\beta}_{1}$.

There were 48 simulations runs in total, and in all but 5 cases the SLS estimators had lower magnitude of bias. In those 5 cases where OLS was better the difference 
in magnitude of bias was at the fourth decimal place. For example in Table 1, the last row shows $\operatorname{Bias}\left(\widehat{\beta}_{0}\right)=-0.0010$ and $\operatorname{Bias}\left(\tilde{\beta}_{0}\right)=-0.0011$, so the difference in magnitude of the biases is only 0.0001. In most case where SLS was superior the difference in biases was large (especially when $\gamma$ was small). For example in the first row of Table 1, we see that $\operatorname{Bias}\left(\widehat{\beta}_{0}\right)=0.2456$ and $\operatorname{Bias}\left(\tilde{\beta}_{0}\right)=-0.0151$. This is not an atypical difference. The magnitude of the bias of the SLS is generally decreasing in $n$, although this is not always the case. However, when the bias does increase as $n$ increases, the increases are small and probably due to sampling variability and not a lack of inconsistency. For example, in Table 3 for the first three rows, $\operatorname{Bias}\left(\tilde{\beta}_{1}\right)=0.0554,0.0132,0.0147$ for $n=500,1000,2000$, respectively. Some general observations concerning the OLS estimates are: a) the OLS biases persist as $n$ gets large, b) the OLS biases are larger when $\gamma$ is smaller (especially for $\widehat{\beta}_{1}, \gamma$ seems to affect the slope parameter more then the intercept), and c) the bias of the OLS intercept is larger in magnitude when $\pi$ is larger (i.e. $\pi$ tends to affect the OLS intercept). It is evident from the simulations that the OLS bias for $\beta_{1}$ is equal to $\beta_{1}(\gamma-1)$ which implies $\operatorname{plim} \hat{\beta}_{1}=\beta_{1} \gamma$. In this case knowing $\gamma$ would lead to a simple consistent estimator of the slope parameter. ${ }^{1}$

The simulations certainly suggest that the SLS estimator generally outperforms the OLS estimator in terms of estimation bias. Figure 2 depicts this bias reduction of SLS over OLS. For the purposes of illustration, this figure contains a single simulation run where $\beta_{0}=0, \beta_{1}=1, \gamma=0.75$, and $\pi=0.10$. The heavy line represents the fitted values for SLS, the medium line are the fitted values for OLS, and the light

\footnotetext{
${ }^{1}$ This is a special case that follows from the assumption of a normal distribution on the $x^{\prime} s$ in a simple regression model. Horrace and Oaxaca (2001) prove the special case and derive the more complicated bias for the OLS estimator for the constant term $\beta_{0}$. An alternative simulation study was performed in which $x_{i}$ had a uniform distribution. The results, available upon request, show that SLS outperforms OLS in terms of finite sample bias of the estimates.
} 
line is the true LPM data generating process. Clearly, the SLS fitted values reflect a smaller bias than the OLS fitted values.

\section{Simulation Study: SLS versus Logit and Probit}

A simulation study was conducted to assess the performance of the SLS estimator against Logit and Probit when the underlying data generation process is LPM. Since logit and probit are commonly employed in dependent variable econometric analysis, a comparison seemed necessary even though it is presumed that the DGP is LPM, implying that logit and probit are mis-specified models. Such a study presents several problems, but the largest problem stems from the fact that probit and logit are nonlinear estimations techniques while SLS and the LPM are linear. Therefore, while bias comparisons of OLS and SLS to the LPM seem natural, bias comparisons of logit and probit to the LPM are not readily forthcoming. Specifically, for logit and probit the estimates of the marginal effect $\beta_{1}$ are functions of the value of $x_{i}$, which raises the question of how one should assess the bias of logit or probit on the LPM, which has a constant slope on $x_{i} \beta \in(0,1)$. Because evaluation of the probit/logit marginal effects at the mean of $x_{i}$ is so popular among empiricists, we initially sought to evaluate the bias of the marginal effects at the mean. However, logit and probit

tended to be highly biased at the mean of $x_{i}$. For example, a typical simulation run is depicted in Figure 3. Here, $\beta_{0}=0, \beta_{1}=1, \gamma=0.75, \pi=0.10$, and the empirical mean of $x_{i}=0.508$. Since $\beta_{1}=1$ and $\beta_{0}=0$, the $\mathrm{x}$-axis in the figure is $x_{i}$. It is clear from the figure that near the mean of $x_{i}$ (about 0.50), probit is upward biased relative to SLS. Therefore, bias at the mean was discarded as a means of evaluating logit and probit on the LPM. However, it is useful to point out that the practice of evaluating the marginal effects at the mean of $x_{i}$ for logit and probit, may lead to biased results when an LPM DGP is suspected.

An alternative evaluation criterion that could be equally applied to SLS and probit or logit is the mean squared error of the predicted probabilities for SLS and probit or 
logit. That is, the simulated LPM data implied a known probability that $y_{i}=1$, which could be compared to the predicted probabilities of SLS, logit and probit. Predicted probability errors for the sample could then be squared, summed and averaged to produce an estimate of the mean squared error of the prediction probabilities. For SLS (and OLS which was ultimately included in the comparison), predicted probabilities greater than 1 where converted to 1 and those less than 0 where converted to 0 before constructing the MSE estimates.

To do this, we selected only two sample sizes of $n=500$ and 1000 . Simulation iterations were again set at 100 to calculate the MSE of the predicted probabilities of the OLS, probit, logit and SLS. Again we assumed that $x_{i}$ was normally distributed. (An alternative simulation study was also performed assuming that $x_{i}$ had a uniform distribution. Parameter values were again selected as $\gamma=0.25$ and $0.75, \pi=0.10$, $0.20, \beta_{0}=-0.50,0.50$, and $\beta_{1}=1.00,2.00$ (as in the simulation study of section 4). This implied 32 simulation runs, the results of which are contained in Tables 5, 6, 7 and 8. In all cases probit and logit are superior to OLS, and SLS is superior to probit and logit. The magnitude of the MSE for all models is generally unaffected by the values of $\beta_{0}$ and $\beta_{1}$; for example the first and third rows of Table 5 are very similar (changing $\beta_{1}$ ceteris paribus) as are the first and fifth (changing $\beta_{0}$ ceteris paribus). Not surprisingly, increasing the sample size tends to decrease the MSE for all models. This is always the case for the consistent SLS procedure (and for probit and logit), but is not always true for the inconsistent OLS procedure. Notice that as $\gamma$ decreases from Table 5 to Table 7, the MSE of OLS tends to significantly increase (by a magnitude of ten-fold), while the MSE of SLS generally does not. This seems to reflect an increased OLS bias associated with smaller $\gamma$ (remember OLS and SLS are equivalent when $\gamma=1$ ). Also notice that as $\gamma$ decreases from Table 5 to Table 7 , the MSE of probit and logit seems to decrease. This is not surprising since logit and probit perform better for extreme values than for median values of $x_{i} \beta$ when the DGP 
is LPM, and since smaller $\gamma$ implies that a greater proportion of the observed $x_{i} \beta$ will be extreme. For median values of $x_{i} \beta$, probit and logit are more highly misspecified, so the larger $\gamma$ of Table 5 produces more $x_{i} \beta$ near the median values and, hence, large MSE for probit and logit. ${ }^{2}$

\section{Concluding Remarks}

Although it is theoretically possible for OLS estimation of the LPM to yield unbiased estimators conditional on the sample, this generally would require fortuitous circumstances. Furthermore, consistency of OLS is shown to be an exceedingly rare occurrence as one would have to accept extraordinary restrictions on the joint distribution of the regressors. Therefore, OLS is frequently a biased estimator and almost always an inconsistent estimator of the LPM. Despite estimation difficulties, the LPM is still frequently used in modeling probabilities. This is partly due to theoretical arguments that justify the linear specification and partly due to the ease of using OLS to estimate the model.

In this paper an alternative estimation strategy has been introduced (SLS) that is fairly easy to implement and offers the promise of significantly reducing the bias from OLS. The conditions under which SLS is consistent are rigorously derived. Monte Carlo simulations with a two parameter LPM support our conjectures about the bias reducing properties of SLS. These simulations also point to the persistence of OLS bias as the sample size increases, which is to be expected when OLS is not consistent. Monte Carlo simulations also suggest that SLS outperforms logit and probit when the DGP is LPM.

Standard errors have not yet been derived for SLS that incorporate the statistical effect of the sequential trimming procedure. It is clear that SLS is not efficient. The

\footnotetext{
${ }^{2}$ Based on simulations in which $x_{i}$ was drawn from a uniform distribution, SLS outperformed OLS, logit, and probit in terms of mean squared error of the predicted probabilities. These results are available upon request.
} 
absence of rogue predictions of $y_{i}$ outside of the unit interval at the outset implies heteroscedastic errors. Conditioning on the final trimmed sample $\widehat{\kappa}_{\gamma}^{(J)}$, one could use a feasible GLS estimator that weights the observations by $1 / \sqrt{\left(\tilde{y}_{i}\right)\left(1-\tilde{y}_{i}\right)}$, where $\tilde{y}_{i}=x_{i} \tilde{\beta}_{i \in \hat{\kappa}_{\gamma}^{(J)}} \in[0,1]$. The estimated standard errors would be obtained in the usual way.

It would be interesting to explore alternative trimming rules for the SLS. For example Seung Ahn (personal communication) has suggested that trimming predicted probabilities outside the interval $[-\omega, 1+\omega]$ for $\omega>0$ may result in a sequential estimator that has lower MSE for parameter estimates. This is the classical bias/efficiency trade-off. Since SLS with $\omega=0$, trims "good" observations with positive probability. SLS with $\omega>0$, may result in a larger final sample size $n_{\gamma}^{(J)}$ (lower variance) at the cost of higher bias of the parameter estimates. Of course this remains to be seen. 


\section{REFERENCES}

Amemiya, Takeshi. "Some Theorems in the Linear Probability Model," International Economic Review, 18(3), October 1977, 645-650.

Currie, Janet and Jonathan Gruber. "Health Insurance Eligibility, Utilization of Medical Care, and Child Health," Quarterly Journal of Economics, May 1996, 431-466.

Greene, William H. Econometric Analysis, 4th ed. (Upper Saddle River, NJ: PrenticeHall, 2000).

Heckman, James J. and James M. Snyder, Jr. "Linear Probability Models of the Demand for Attributes With an Empirical Application to Estimating the Preferences of Legislators," Rand Journal of Economics, 28(0), 1997, S142S189.

Horrace, William C. and Ronald L. Oaxaca. "Distributional Assumptions and OLS Bias in the LPM," mimeo, May 2001.

Kmenta, Jan. Elements of Econometrics, 2nd ed. (New York: Macmillan, 1986).

Lucking-Reiley, David. "Magic on the Internet: Evidence from Field Experiments on Reserve Prices in Auctions," mimeo, February 1999.

Lukashin, Youri Pavlovich. "Econometric Analysis of Managers' Judgements on the Determinants of the Financial Situation in Russia," Economics of Planning, $33,2000,85-101$.

Fairlie, Robert and William A. Sundstrom. "The Emergence, Persistence, and Recent Widening of the Racial Unemployment Gap," Industrial and Labor Relations Review, 52(2), January 1999, 252-270. 
Rosenthal, R.W. "A Bounded-Rationality Approach to the Study of Noncooperative Games," 18, 1989, 273-292. 
Table 1. $\gamma=0.75, \pi=0.10$

\begin{tabular}{||r|r|r||r|r||r|r||}
\hline \hline$\beta_{0}$ & $\beta_{1}$ & $n$ & $\operatorname{Bias}\left(\widehat{\beta}_{0}\right)$ & $\operatorname{Bias}\left(\tilde{\beta}_{0}\right)$ & $\operatorname{Bias}\left(\widehat{\beta}_{1}\right)$ & $\operatorname{Bias}\left(\tilde{\beta}_{1}\right)$ \\
\hline \hline \multirow{3}{*}{-0.50} & \multirow{2}{*}{1.00} & 500 & 0.2456 & -0.0151 & -0.2470 & 0.0139 \\
\cline { 3 - 7 } & & 1000 & 0.2486 & -0.0089 & -0.2470 & 0.0112 \\
\cline { 3 - 7 } & & 2000 & 0.2489 & -0.0082 & -0.2501 & 0.0072 \\
\hline \hline \multirow{3}{*}{0.50} & \multirow{2}{*}{2.00} & 500 & 0.2523 & -0.0072 & -0.5022 & 0.0204 \\
\cline { 3 - 7 } & & 1000 & 0.2485 & -0.0085 & -0.4983 & 0.0158 \\
\cline { 3 - 7 } & & 2000 & 0.2499 & -0.0041 & -0.4997 & 0.0082 \\
\hline \hline \multirow{2}{*}{0.50} & \multirow{2}{*}{5.00} & 500 & 0.0000 & -0.0005 & -0.2476 & 0.0072 \\
\cline { 3 - 7 } & & 1000 & 0.0003 & 0.0014 & -0.2516 & 0.0032 \\
\cline { 3 - 7 } & 2000 & 0.0003 & 0.0001 & -0.2504 & -0.0001 \\
\hline \hline \multirow{3}{*}{0.50} & 500 & -0.0018 & -0.0024 & -0.4956 & 0.0257 \\
\cline { 3 - 7 } & & 1000 & 0.0009 & 0.0010 & -0.4969 & 0.0079 \\
\cline { 3 - 7 } & 2000 & -0.0010 & -0.0011 & -0.5090 & -0.0124 \\
\hline \hline
\end{tabular}


Table 2. $\gamma=0.75, \pi=0.20$

\begin{tabular}{||r|r|r||r|r||r|r||}
\hline \hline$\beta_{0}$ & $\beta_{1}$ & $n$ & $\operatorname{Bias}\left(\widehat{\beta}_{0}\right)$ & $\operatorname{Bias}\left(\tilde{\beta}_{0}\right)$ & $\operatorname{Bias}\left(\widehat{\beta}_{1}\right)$ & $\operatorname{Bias}\left(\tilde{\beta}_{1}\right)$ \\
\hline \hline \multirow{3}{*}{-0.50} & \multirow{2}{*}{1.00} & 500 & 0.2576 & -0110 & -0.2487 & 0.0173 \\
\cline { 3 - 7 } & & 1000 & 0.2502 & -0121 & -0.2480 & 0.0096 \\
\cline { 3 - 7 } & & 2000 & 0.2522 & -0018 & -0.2497 & -0.0002 \\
\hline \hline \multirow{3}{*}{0.50} & \multirow{2}{*}{2.00} & 500 & 0.2626 & -0066 & -0.5103 & 0.0208 \\
\cline { 3 - 7 } & & 1000 & 0.2523 & -0014 & -0.4981 & 0.0015 \\
\cline { 3 - 7 } & & 2000 & 0.2516 & -0036 & -0.4963 & 0.0068 \\
\hline \hline \multirow{2}{*}{0.50} & \multirow{2}{*}{500} & 500 & 0.0030 & -0012 & -0.2443 & 0.0160 \\
\cline { 3 - 7 } & & 1000 & 0.0041 & -0001 & -0.2487 & 0.0092 \\
\cline { 3 - 7 } & 2.00 & 500 & 0.0038 & -0002 & -0.2473 & 0.0110 \\
\cline { 3 - 7 } & 1000 & 0.0022 & -0.0029 & -0.4901 & 0.0237 \\
\cline { 3 - 7 } & 2000 & 0.0063 & 0.0023 & -0.5042 & -0.0048 \\
\hline \hline
\end{tabular}


Table 3. $\gamma=0.25, \pi=0.10$

\begin{tabular}{||r|r|r||r|r||r|r||}
\hline \hline$\beta_{0}$ & $\beta_{1}$ & $n$ & $\operatorname{Bias}\left(\widehat{\beta}_{0}\right)$ & $\operatorname{Bias}\left(\tilde{\beta}_{0}\right)$ & $\operatorname{Bias}\left(\widehat{\beta}_{1}\right)$ & $\operatorname{Bias}\left(\tilde{\beta}_{1}\right)$ \\
\hline \hline \multirow{3}{*}{-0.50} & \multirow{2}{*}{1.00} & 500 & 0.6916 & -0.0533 & -0.7498 & 0.0554 \\
\cline { 3 - 7 } & & 1000 & 0.6920 & -0.0140 & -0.7498 & 0.0132 \\
\cline { 3 - 7 } & & 2000 & 0.6934 & -0.0122 & -0.7498 & 0.0147 \\
\hline \hline \multirow{3}{*}{0.50} & \multirow{2}{*}{2.00} & 500 & 0.6930 & -0.0456 & -1.4993 & 0.0889 \\
\cline { 3 - 7 } & & 1000 & 0.6930 & -0.0095 & -1.5006 & 0.0192 \\
\cline { 3 - 7 } & & 2000 & 0.6937 & -0.0058 & -1.4990 & 0.0184 \\
\hline \hline \multirow{2}{*}{0.50} & \multirow{2}{*}{500} & 500 & -0.0610 & 0.0093 & -0.7515 & 0.0391 \\
\cline { 3 - 7 } & & 1000 & -0.0574 & 0.0014 & -0.7501 & 0.0100 \\
\cline { 3 - 7 } & 2000 & -0.0579 & 0.0014 & -0.7502 & 0.0108 \\
\hline \hline \multirow{3}{*}{0.50} & 500 & -0.0535 & 0.0071 & -1.4970 & 0.0838 \\
\cline { 3 - 7 } & 1000 & -0.0569 & 0.0022 & -1.4985 & 0.0339 \\
\cline { 3 - 7 } & 2000 & -0.0591 & 0.0023 & -1.5014 & 0.0096 \\
\hline \hline
\end{tabular}


Table 4. $\gamma=0.25, \pi=0.20$

\begin{tabular}{||r|r|r||r|r||r|r||}
\hline$\beta_{0}$ & $\beta_{1}$ & $n$ & $\operatorname{Bias}\left(\widehat{\beta}_{0}\right)$ & $\operatorname{Bias}\left(\tilde{\beta}_{0}\right)$ & $\operatorname{Bias}\left(\widehat{\beta}_{1}\right)$ & $\operatorname{Bias}\left(\tilde{\beta}_{1}\right)$ \\
\hline \hline \multirow{3}{*}{-0.50} & \multirow{2}{*}{1.00} & 500 & 0.7371 & -0.0301 & -0.7488 & 0.0327 \\
\cline { 3 - 7 } & & 1000 & 0.7354 & -0.0116 & -0.7493 & 0.0084 \\
\cline { 3 - 7 } & & 2000 & 0.7382 & -0.0155 & -0.7498 & 0.0185 \\
\hline \hline \multirow{3}{*}{-0.50} & \multirow{2}{*}{0.00} & 500 & 0.7344 & -0.0430 & -1.5009 & 0.0775 \\
\cline { 3 - 7 } & & 1000 & 0.7368 & -0.0221 & -1.5000 & 0.0409 \\
\cline { 3 - 7 } & & 2000 & 0.7362 & -0.0035 & -1.4985 & 0.0071 \\
\hline \hline \multirow{2}{*}{0.50} & \multirow{2}{*}{0.00} & 500 & -0.0135 & -0.0032 & -0.7491 & 0.0270 \\
\cline { 3 - 7 } & & 1000 & -0.0119 & 0.0050 & -0.7497 & 0.0421 \\
\cline { 3 - 7 } & 2000 & -0.0131 & -0.0002 & -0.7501 & 0.0146 \\
\hline \hline \multirow{2}{*}{0.50} & \multirow{2}{*}{2.00} & 500 & -0.0121 & 0.0035 & -1.4988 & 0.0861 \\
\cline { 3 - 7 } & & 1000 & -0.0111 & 0.0024 & -1.5001 & 0.0343 \\
\cline { 3 - 7 } & 2000 & -0.0135 & 0.0007 & -1.4989 & 0.0391 \\
\hline \hline
\end{tabular}


Table 5. $\gamma=0.75, \pi=0.10$

\begin{tabular}{||r|r|r||r|r||r|r||}
\hline \hline$\beta_{0}$ & $\beta_{1}$ & $n$ & $M S E\left(\widehat{y}_{\text {OLS }}\right)$ & $M S E\left(\widehat{y}_{\text {probit }}\right)$ & $M S E\left(\widehat{y}_{\text {Logit }}\right)$ & $M S E\left(\widehat{y}_{S L S}\right)$ \\
\hline \hline \multirow{2}{*}{-0.50} & \multirow{2}{*}{1.00} & 500 & 0.00407 & 0.00150 & 0.00193 & 0.00061 \\
\cline { 3 - 7 } & & 1000 & 0.00418 & 0.00131 & 0.00172 & 0.00034 \\
\hline \hline \multirow{2}{*}{-0.50} & \multirow{2}{*}{2.00} & 500 & 0.00432 & 0.00137 & 0.00176 & 0.00053 \\
\cline { 3 - 7 } & & 1000 & 0.00410 & 0.00118 & 0.00158 & 0.00024 \\
\hline \hline \multirow{2}{*}{0.50} & \multirow{2}{*}{1.00} & 500 & 0.00419 & 0.00172 & 0.00217 & 0.00085 \\
\cline { 3 - 7 } & & 1000 & 0.00424 & 0.00121 & 0.00161 & 0.00030 \\
\hline \hline \multirow{2}{*}{0.50} & \multirow{2}{*}{2.00} & 500 & 0.00427 & 0.00153 & 0.00195 & 0.00063 \\
\cline { 3 - 7 } & & 1000 & 0.00417 & 0.00128 & 0.00169 & 0.00033 \\
\hline \hline
\end{tabular}

Table 6. $\quad \gamma=0.75, \pi=0.20$

\begin{tabular}{||r|r|r||r|r||r|r||}
\hline \hline$\beta_{0}$ & $\beta_{1}$ & $n$ & $M S E\left(\widehat{y}_{\text {OLS }}\right)$ & $M S E\left(\widehat{y}_{\text {probit }}\right)$ & $M S E\left(\widehat{y}_{\text {Logit }}\right)$ & $M S E\left(\widehat{y}_{S L S}\right)$ \\
\hline \hline \multirow{2}{*}{-0.50} & \multirow{2}{*}{1.00} & 500 & 0.00417 & 0.00152 & 0.00194 & 0.00063 \\
\cline { 3 - 7 } & & 1000 & 0.00406 & 0.00124 & 0.00165 & 0.00030 \\
\hline \hline \multirow{2}{*}{-0.50} & \multirow{2}{*}{2.00} & 500 & 0.00419 & 0.00152 & 0.00196 & 0.00063 \\
\cline { 3 - 7 } & & 1000 & 0.00408 & 0.00131 & 0.00172 & 0.00035 \\
\hline \hline \multirow{2}{*}{0.50} & \multirow{2}{*}{0.00} & 500 & 0.00417 & 0.00141 & 0.00182 & 0.00051 \\
\cline { 3 - 7 } & & 1000 & 0.00397 & 0.00130 & 0.00171 & 0.00032 \\
\hline \hline \multirow{2}{*}{0.50} & \multirow{2}{*}{2.00} & 500 & 0.00417 & 0.00156 & 0.00199 & 0.00060 \\
\cline { 3 - 7 } & & 1000 & 0.00419 & 0.00118 & 0.00157 & 0.00029 \\
\hline \hline
\end{tabular}


Table 7. $\gamma=0.25, \pi=0.10$

\begin{tabular}{||r|r|r||r|r||r|r||}
\hline \hline$\beta_{0}$ & $\beta_{1}$ & $n$ & $M S E\left(\widehat{y}_{\text {OLS }}\right)$ & $M S E\left(\widehat{y}_{\text {probit }}\right)$ & $M S E\left(\widehat{y}_{\text {Logit }}\right)$ & $M S E\left(\widehat{y}_{S L S}\right)$ \\
\hline \hline \multirow{2}{*}{-0.50} & \multirow{2}{*}{1.00} & 500 & 0.03653 & 0.00095 & 0.00124 & 0.00061 \\
\cline { 3 - 7 } & & 1000 & 0.03623 & 0.00068 & 0.00095 & 0.00026 \\
\hline \hline \multirow{2}{*}{-0.50} & \multirow{2}{*}{2.00} & 500 & 0.03637 & 0.00085 & 0.00113 & 0.00049 \\
\cline { 3 - 7 } & & 1000 & 0.03630 & 0.00074 & 0.00103 & 0.00030 \\
\hline \hline \multirow{2}{*}{0.50} & \multirow{2}{*}{1.00} & 500 & 0.03636 & 0.00097 & 0.00126 & 0.00069 \\
\cline { 3 - 7 } & & 1000 & 0.03623 & 0.00068 & 0.00095 & 0.00026 \\
\hline \hline \multirow{2}{*}{0.50} & \multirow{2}{*}{2.00} & 500 & 0.03628 & 0.00081 & 0.00108 & 0.00046 \\
\cline { 3 - 7 } & & 1000 & 0.03614 & 0.00076 & 0.00105 & 0.00030 \\
\hline \hline
\end{tabular}

Table 8. $\gamma=0.25, \pi=0.20$

\begin{tabular}{||r|r|r||r|r||r|r||}
\hline \hline$\beta_{0}$ & $\beta_{1}$ & $n$ & $M S E\left(\widehat{y}_{\text {OLS }}\right)$ & $M S E\left(\widehat{y}_{\text {probit }}\right)$ & $M S E\left(\widehat{y}_{\text {Logt }}\right)$ & $M S E\left(\widehat{y}_{S L S}\right)$ \\
\hline \hline \multirow{2}{*}{0.50} & \multirow{2}{*}{1.00} & 500 & 0.04090 & 0.00090 & 0.00118 & 0.00057 \\
\cline { 3 - 7 } & & 1000 & 0.04080 & 0.00072 & 0.00099 & 0.00028 \\
\hline \hline \multirow{2}{*}{-0.50} & \multirow{2}{*}{0.00} & 500 & 0.04122 & 0.00092 & 0.00120 & 0.00049 \\
\cline { 3 - 7 } & & 1000 & 0.04073 & 0.00076 & 0.00103 & 0.00031 \\
\hline \hline \multirow{2}{*}{0.50} & \multirow{2}{*}{1.00} & 500 & 0.04076 & 0.00094 & 0.00123 & 0.00059 \\
\cline { 3 - 7 } & & 1000 & 0.04098 & 0.00068 & 0.00095 & 0.00026 \\
\hline \hline \multirow{2}{*}{0.50} & \multirow{2}{*}{2.00} & 500 & 0.04117 & 0.00098 & 0.00128 & 0.00067 \\
\cline { 3 - 7 } & & 1000 & 0.04108 & 0.00069 & 0.00097 & 0.00030 \\
\hline \hline
\end{tabular}




\section{IZA Discussion Papers}

\begin{tabular}{|c|c|c|c|c|}
\hline No. & Author(s) & Title & Area & Date \\
\hline 688 & $\begin{array}{l}\text { I. Haouas } \\
\text { M. Yagoubi } \\
\text { A. Heshmati }\end{array}$ & $\begin{array}{l}\text { The Impacts of Trade Liberalization on } \\
\text { Employment and Wages in Tunisian Industries }\end{array}$ & 4 & $01 / 03$ \\
\hline 689 & $\begin{array}{l}\text { I. Haouas } \\
\text { A. Heshmati }\end{array}$ & $\begin{array}{l}\text { The Effects of Union Wage-Settings on Firms' } \\
\text { Production Factor Decisions }\end{array}$ & 5 & $01 / 03$ \\
\hline 690 & M. Hagedorn & Contracting with Private Information & 1 & $01 / 03$ \\
\hline 691 & $\begin{array}{l}\text { B. Petrongolo } \\
\text { C. A. Pissarides }\end{array}$ & Scale Effects in Markets with Search & 3 & $01 / 03$ \\
\hline 692 & $\begin{array}{l}\text { T. E. Smith } \\
\text { Y. Zenou }\end{array}$ & $\begin{array}{l}\text { Spatial Mismatch, Search Effort and Urban } \\
\text { Spatial Structure }\end{array}$ & 3 & $01 / 03$ \\
\hline 693 & $\begin{array}{l}\text { L. Gobillon } \\
\text { H. Selod } \\
\text { Y. Zenou }\end{array}$ & $\begin{array}{l}\text { Spatial Mismatch: From the Hypothesis to the } \\
\text { Theories }\end{array}$ & 3 & $01 / 03$ \\
\hline 694 & E. S. Prasad & $\begin{array}{l}\text { What Determines the Reservation Wages of } \\
\text { Unemployed Workers? New Evidence from } \\
\text { German Micro Data }\end{array}$ & 3 & $01 / 03$ \\
\hline 695 & $\begin{array}{l}\text { S. Alan } \\
\text { T. F. Crossley } \\
\text { P. Grootendorst } \\
\text { M. R. Veall }\end{array}$ & $\begin{array}{l}\text { Out-of-Pocket Prescription Drug Expenditures } \\
\text { and Public Prescription Drug Programs }\end{array}$ & 3 & $01 / 03$ \\
\hline 696 & R. Schettkat & $\begin{array}{l}\text { Institutions in the Economic Fitness Landscape: } \\
\text { What Impact Do Welfare State Institutions Have } \\
\text { on Economic Performance? }\end{array}$ & 3 & $01 / 03$ \\
\hline 697 & R. Schettkat & $\begin{array}{l}\text { Differences in US-German Time-Allocation: } \\
\text { Why Do Americans Work Longer Hours than } \\
\text { Germans? }\end{array}$ & 5 & $01 / 03$ \\
\hline 698 & $\begin{array}{l}\text { T. M. Andersen } \\
\text { J. R. Skaksen }\end{array}$ & $\begin{array}{l}\text { Product Market Integration, Comparative } \\
\text { Advantages and Labour Market Performance }\end{array}$ & 2 & $01 / 03$ \\
\hline 699 & $\begin{array}{l}\text { D. Margolis } \\
\text { V. Simonnet }\end{array}$ & $\begin{array}{l}\text { Educational Track, Networks and Labor Market } \\
\text { Outcomes }\end{array}$ & 2 & $01 / 03$ \\
\hline 700 & T. K. Bauer & $\begin{array}{l}\text { Flexible Workplace Practices and Labor } \\
\text { Productivity }\end{array}$ & 1 & $01 / 03$ \\
\hline 701 & $\begin{array}{l}\text { L. Goerke } \\
\text { J. B. Madsen }\end{array}$ & $\begin{array}{l}\text { Earnings-Related Unemployment Benefits in a } \\
\text { Unionised Economy }\end{array}$ & 3 & $01 / 03$ \\
\hline 702 & $\begin{array}{l}\text { M. Ayhan Kose } \\
\text { E. S. Prasad } \\
\text { M. E. Terrones }\end{array}$ & $\begin{array}{l}\text { How Does Globalization Affect the } \\
\text { Synchronization of Business Cycles? }\end{array}$ & 2 & $01 / 03$ \\
\hline 703 & $\begin{array}{l}\text { W. C. Horrace } \\
\text { R. L. Oaxaca }\end{array}$ & $\begin{array}{l}\text { New Wine in Old Bottles: A Sequential } \\
\text { Estimation Technique for the LPM }\end{array}$ & 6 & $01 / 03$ \\
\hline
\end{tabular}

An updated list of IZA Discussion Papers is available on the center's homepage www.iza.org. 